\title{
Paraneoplastic Syndromes
}

National Institute of Neurological Disorders and Stroke (NINDS)

\section{Source}

National Institute of Neurological Disorders and Stroke (NINDS). Paraneoplastic

Syndromes Information Page.

Paraneoplastic syndromes are a group of rare disorders that are triggered by an abnormal immune system response to a cancerous tumor known as a "neoplasm." Paraneoplastic syndromes are thought to happen when cancer-fighting antibodies or white blood cells (known as T cells) mistakenly attack normal cells in the nervous system. These disorders typically affect middle-aged to older people and are most common in individuals with lung, ovarian, lymphatic, or breast cancer. Neurologic symptoms generally develop over a period of days to weeks and usually occur prior to the tumor being discovered. These symptoms may include difficulty in walking or swallowing, loss of muscle tone, loss of fine motor coordination, slurred speech, memory loss, vision problems, sleep disturbances, dementia, seizures, sensory loss in the limbs, and vertigo or dizziness. Paraneoplastic syndromes include Lambert-Eaton myasthenic syndrome, stiff-person syndrome, encephalomyelitis, myasthenia gravis, cerebellar degeneration, limbic or brainstem encephalitis, neuromyotonia, opsoclonus, and sensory neuropathy. 